\title{
Less is more approach: basic variable neighborhood search for the obnoxious $p$-median problem
}

\author{
Nenad Mladenovića ${ }^{a, b}$, Abdulaziz Alkandaric ${ }^{\mathrm{c}}$, Jun Pei ${ }^{\mathrm{d}, \mathrm{e}}$, Raca Todosijević ${ }^{\mathrm{f}, \mathrm{g}}$ and \\ Panos M. Pardalos ${ }^{\mathrm{h}}$ \\ ${ }^{a}$ Emirates College of Technology, Abu Dhabi, United Arab Emirates \\ ${ }^{\mathrm{b}}$ Ural Federal University, Yekaterinburg, Russia \\ ${ }^{\mathrm{C}}$ College of Technological Studies, Public Authority for Applied Education and Training, Kuwait City, Kuwait \\ ${ }^{\mathrm{d}}$ School of Management, Hefei University of Technology, Anhui Sheng, China \\ ${ }^{\mathrm{e}}$ Key Laboratory of Process Optimization and Intelligent Decision-Making, Ministry of Education, Hefei, China \\ ${ }^{\mathrm{f}}$ LAMIH UMR CNRS 8201, Université Polytechnique Hauts-de-France, 59313 Valenciennes Cedex 9, Famars, France \\ ${ }^{\mathrm{g}}$ Mathematical Institute of the Serbian Academy of Science and Arts, Kneza Mihaila 36, 11000 Belgrade, Serbia \\ ${ }^{\mathrm{h}}$ Department of Industrial and Systems Engineering, Faculty of Engineering, University of Florida, Gainesville, FL \\ 32611-6595, USA \\ E-mail:nenadmladenovic12@gmail.com [Mladenović]; abdl_alkandari@hotmail.com [Alkandari]; \\ feiyijun198612@126.com [Pei]; racatodosijevic@gmail.com [Todosijević]; pardalos@ufl.edu [Pardalos]
}

Received 21 December 2017; received in revised form 10 February 2019; accepted 11 February 2019

\begin{abstract}
The goal of the less is more approach (LIMA) for solving optimization problems that has recently been proposed in Mladenovic et al. (2016) is to find the minimum number of search ingredients that make a heuristic more efficient than the currently best. In this paper, LIMA is successfully applied to solve the obnoxious $p$-median problem (OpMP). More precisely, we developed a basic variable neighborhood search for solving the OpMP, where the single search ingredient, the interchange neighborhood structure, is used. We also propose a new simple local search strategy for solving facility location problems, within the interchange neighborhood structure, which is in between the usual ones: first improvement and best improvement strategies. We call it facility best improvement local search. On the basis of experiments, it appeared to be more efficient and effective than both first and best improvement. According to the results obtained on the benchmark instances, our heuristic turns out to be highly competitive with the existing ones, establishing new state-ofthe-art results. For example, four new best-known solutions and 133 ties are claimed in testing the set with 144 instances.
\end{abstract}

Keywords: heuristic; less is more; obnoxious location; heap data structure; facility best improvement

(C) 2019 The Authors.

International Transactions in Operational Research () 2019 International Federation of Operational Research Societies Published by John Wiley \& Sons Ltd, 9600 Garsington Road, Oxford OX4 2DQ, UK and 350 Main St, Malden, MA02148, USA. 


\section{Introduction}

Obnoxious location problems are those that take into account the so-called obnoxious or semiobnoxious effects. These effects often occur when it is desired that a facility be located as far as possible from an inhabited center (obnoxious effect) or when a facility cannot be located far enough, although its closeness can have immediate disturbing or dangerous effects (semiobnoxious effect). Location problems with obnoxious effect arise when locating nuclear power plants, chemical plants, and dump sites, while examples of location problems with semiobnoxious effect include locating airports, recycle plants, and so on.

The first obnoxious location model in the literature is given by Church and Garfinkel (1978) who considered location of a facility in a network. Since that time, researchers studied many variants of obnoxious location problems. The recent literature review on existing variants and solution techniques developed for solving obnoxious problems may be found in Colmenar et al. (2016).

In this paper, we focus on the obnoxious $p$-median problem (OpMP). Given a set $J$ of possible facility locations and a set of customers $I$, as well as the distance $d_{i j}$ between each customer $i \in I$ and facility location $j \in J$, the OpMP consists in choosing $p$ facility locations from the given set so that the sum of the distances between each customer and its nearest facility is maximized. Formally, the problem may be stated as:

$$
\max \sum_{i \in I} \min \left\{d_{i j}: j \in S\right\}
$$

subject to

$$
S \subset J,|S|=p .
$$

The OpMP may be stated as a mixed integer program using binary variables $y_{j}, j \in J$, which indicate whether the facility location $j$ is chosen or not, and binary variables $x_{i j}, i \in I, j \in J$, which indicate whether the client $i$ is allocated to the facility location $j$ or not. The resulting model is as follows (Belotti et al., 2007):

$$
\max \sum_{i \in I} \sum_{j \in J} d_{i j} x_{i j}
$$

subject to

$$
\begin{aligned}
& \sum_{j \in J} y_{j}=p, \\
& x_{i j} \leq y_{j} \forall i \in I, j \in J, \\
& y_{j}+\sum_{k \in S(i, j)} x_{i k} \leq 1 \forall i \in I, j \in J, \\
& y_{j}, x_{i j} \in\{0,1\} \forall i \in I, j \in J,
\end{aligned}
$$


where $S(i, j)$ is the set of facilities more distant than $j$ from the client $i$, that is,

$$
S(i, j)=\left\{k \in J \mid\left(d_{i k}>d_{i j}\right) \vee\left(d_{i k}=d_{i j} \wedge k>j\right)\right\} .
$$

The meaning of constraints in the model is as follows. Constraint (4) ensures that exactly $p$ facility locations are chosen, while constraint (5) guarantees that customers are allocated only to open facilities. Finally, constraints (6) assign each customer to the closest open facility. Note that the constraints that assign one facility to each customer, that is, $\sum_{j \in J} x_{i j}=1 \forall i \in I$, are included in constraints (6), where sign $\leq$ is used instead of equality because of the non-negativity of distances $d_{i j}$.

Unlike classical $p$-median problem, which is well studied in the literature (see, e.g., Mladenović et al., 2007), the obnoxious $p$-median gained relatively few attention. Since its introduction in the 1990s (Eiselt and Laporte, 1995; Welch and Salhi, 1997; Cappanera, 1999), just a few methods have been proposed to tackle this NP-hard problem (Tamir, 1991). Belotti et al. (2007) developed a branch and cut (B\&C) algorithm coupled with a Tabu search (TS), while recently Colmenar et al. (2016) proposed a greedy randomized adaptive search procedure (GRASP) based heuristic, which uses a filtering mechanism in order to avoid applying a local search on low-quality constructed solutions. More recently, Herrán et al. (2020) proposed a parallel heuristic based on variable neighborhood search (VNS).

In this paper, we follow the recent heuristic approach, that is, less is more approach (LIMA), to solve optimization problems (Mladenović et al., 2016; Brimberg et al., 2017). LIMA's main idea is to find the minimum number of search ingredients when solving a particular optimization problem, which would make some heuristic more efficient than the currently best in the literature. In other words, the goal is to make a heuristic as simple as possible, but at the same time, more effective and efficient than the current state-of-the-art heuristic. We believe that discovering the simple and userfriendly heuristic for some particular problem, which is at the same time more effective and efficient than the others, represents a contribution to the science (rather than just combining methods without having good computational results, only to claim new methodological contribution, as it is often done in some hybrid approaches). By minimizing the number of ingredients in the search, it is much easier to get the answer to the typically made question "why heuristic is working well?". Following the LIMA's idea, we propose a basic VNS heuristic for solving the OpMP. The proposed heuristic uses only one neighborhood structure in both intensification and diversification phases of VNS.

The second contribution of this paper is our new simple local search strategy for solving facility location problems, that is in between usual ones (i.e., the first improvement and the best improvement). We call it "facility best improvement local search."

The usually used data structures for solving facility location problems are proposed in Hansen and Mladenović (1997) and Resende and Werneck (2003) (for the survey, see also Mladenović et al., 2007). In this paper, we propose new simple data structure for saving and updating a solution. It is based on heap data structure. Its complexity is analyzed.

With our Basic VNS that contains new results and contributions mentioned above, we succeed to obtain remarkable results on the benchmark instances from the literature. The proposed approach yet quite simple outperforms existing single-thread state-of-the-art approaches, that is, TS and GRASP. In addition, it is quite competitive in comparison with the parallel VNS (P-VNS). For example, four new best-known solutions (BKS) and 133 ties are claimed in testing the set with 144 instances. Such outcome indicates that sometimes less may yield more. 
The rest of the paper is organized as follows. In Section 2, we provide thorough description of the proposed approach, whereas in Section 3, we assess the merit of the proposed approach. Finally, in Section 4, we draw some concluding remarks and indicate possible future research directions.

\section{Basic variable neighborhood search for the OpMP}

VNS is a flexible framework for building heuristics for approximate solution of optimization problems. It was introduced by Mladenović and Hansen (1997) and its main idea is to systematically change neighborhood structures during the search for an optimal (or near-optimal) solution. This idea comes from the following properties: (i) a local optimum relative to one neighborhood structure is not necessarily a local optimum for another neighborhood structure; (ii) a global optimum is a local optimum with respect to all neighborhood structures. The first property is usually exploited by using multiple local searches in the improvement step as in variable neighborhood descent (see, e.g., Hansen et al., 2017; Mjirda et al., 2017; Duarte et al., 2018). The second property suggests using several neighborhoods, if the found local optima are of poor quality.

The basic variant of VNS (called Basic VNS) consists of executing, alternately, one local search procedure (used to improve a solution) and one so-called shaking procedure (used to hopefully resolve local minima traps), together with the neighborhood change step. The whole process is iterated until a predefined stopping condition (e.g., maximum number of iterations or maximum CPU time) is met. Many variants of VNS have been derived from the Basic VNS scheme and applied to different optimization problems (for a survey, see, e.g., Hansen et al., 2010; for recent publications, see Irawan et al., 2020; Janković et al., 2017; Pinto et al., 2020; Brimberg et al., 2019; Chagas et al., 2020; Gruler et al., 2020).

\subsection{Basic VNS}

For solving the OpMP, we developed a heuristic that follows the rules of Basic VNS. Before providing more details of our heuristic, we will describe the solution representation for the OpMP. The solution of the OpMP is represented as a set $S$ of $p$-chosen facilities because once we know the chosen facilities, each customer is directly assigned to the closest chosen facility. The steps of our Basic VNS for OpMP are given in Algorithm 1. It starts by creating an initial solution at random (see Algorithm 2), which is also the simplest possible way to start. Namely, the initial solution is formed by choosing $p$ facilities from the set $J$ at random. Such a solution is improved through main VNS loop, which includes executing shaking, local search, and neighborhood change procedures, one after another (see steps 5-9). In both shaking and local search steps, the same Interchange neighborhood structure is used, based on closing an open facility in $S$ and opening facility that does not belong to $S$. Formally, the interchange neighborhood of a given solution $S$ is defined as:

$$
\text { Interchange }(S)=\left\{S^{\prime} \subset J|| S \cap S^{\prime}|=| S|-1,| S^{\prime}|=| S \mid=p\right\} .
$$



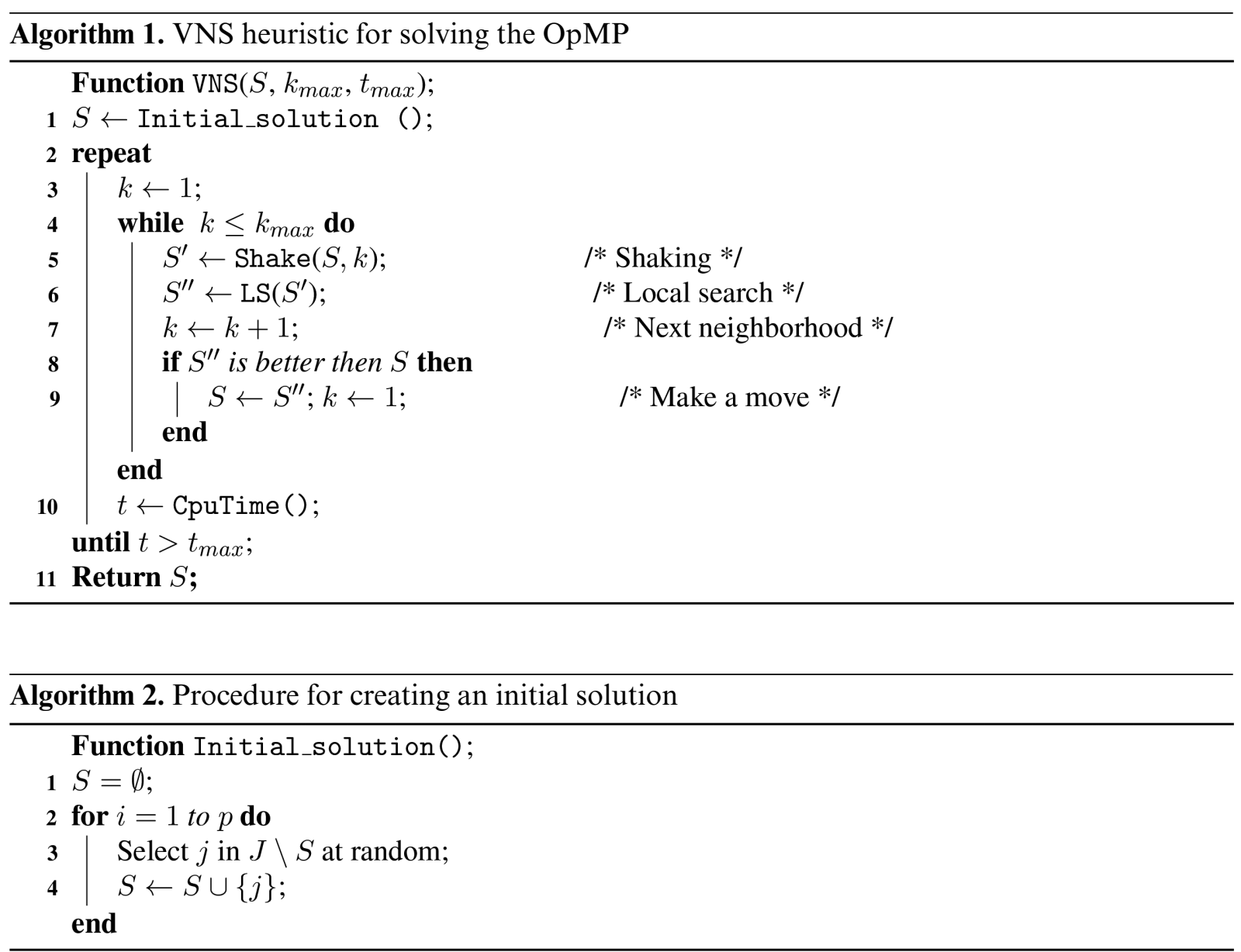

\subsection{Heap data structure}

In order to efficiently evaluate the objective function value of each solution in that neighborhood, for each customer $i$ we maintain a heap data structure $h(i)$, containing facilities in the solution $S$ ordered according to their closeness to the client $i$, and a $|I| \times|J|$ matrix index whose each entry index $(i, j) j \in S$ corresponds to the index of the facility $j$ in the heap $h(i)$. Thanks to such data structure, evaluating each solution $S^{\prime}$ requires $O(|I|)$ operations. Namely, if the first facility in the heap $h(i)$ is replaced by a facility $i^{\prime}$, new facility closest to the customer $i$ becomes facility $i^{\prime}$, the second or the third facility in the heap $h(i)$. Otherwise, new facility closest to the customer $i$ is either the first facility in the heap or newly opened facility $i^{\prime}$. After executing one interchange move, updating data structures $h(i)$ and index requires $O(|I| \log (p))$ operations. Such complexity is due to data structure index, which enables us to detect the position of each facility to be closed by a move in the constant time in each heap $h(i)$.

The data structure used in Colmenar et al. (2016) is based on maintaining two matrices. Such data structure enables move evaluation in the complexity $O(|I| \log (|J|))+O(|I|)=O(|I| \log (|J|))$, 
while our heap data structure enables move evaluation in $O(|I| \log (p))$. Therefore, our heap data structure may be considerably better than the data structure presented in Colmenar et al. (2016), since the value $p$ may be significantly less than $|J|$.

\subsection{Shaking}

The shaking routine is also the simplest possible. It has two formal parameters: the solution $S$ and the neighborhood index $k$ that determines the number of interchange steps performed. In other words, each iteration consists in choosing a random facility to go into $S$, replacing the other, also taken at random, that should go out. Obviously, it could happen that the same facility is chosen two times, that is, it is first removed from $S$ and then returned back to $S$. It is clear that we could easily forbid such cases. However, for the sake of simplicity, we did not do that. It means that solutions from $N_{s}(S)$ are generated, where $s \leq k$.

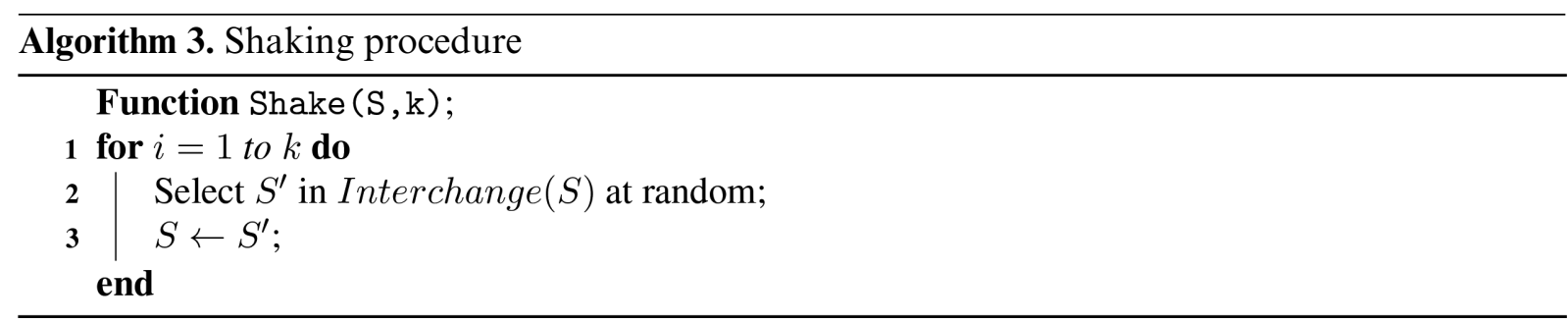

As has already been described, our Basic VNS uses one neighborhood structure within both key steps of VNS that are iterated: improvement procedure and shaking procedure (see steps 5 and 6). Moreover, the Move or not step is also the simplest possible (steps 6-9): move is made only if the better solution in the local search (step 6) is found. In addition, we set $k_{\max }$ to $p$ in all testing, thus reducing the number of formal parameters of Basic VNS to single one, that is, $t_{\max }$, the limit on CPU time. So, our Basic VNS is quite simple and meets the main requirements of a heuristic: simplicity and user-friendliness.

\section{Computational results}

The proposed Basic VNS is coded in $\mathrm{C}++$ language and executed on a machine with an Intel Xeon E7 $4820 \mathrm{CPU}(2.00 \mathrm{GHz})$ and $16 \mathrm{~GB}$ of RAM. For testing purposes, the set of 144 benchmark instances from the literature have been used. These instances were created by Belotti et al. (2007), transforming the benchmark instances for the classical $p$-median problem publicly available at www.people.brunel.ac.uk/ mastjjb/jeb/orlib/pmedinfo.html. In particular, 24 instances (from pmed17 to pmed40) of the $p$-median problem were transformed into 72 instances for the OpMP. The transformation of each instance was performed by the procedure described in Belotti et al. (2007). Given the original instance with $n$ nodes, the procedure generates three instances for the OpMP by considering three values of $p:\lfloor n / 2\rfloor,\lfloor n / 4\rfloor$ and $\lfloor n / 8\rfloor$ and selecting $n / 2$ nodes at random to constitute the set of clients and declaring the remaining $n / 2$ nodes to be the facilities. Recently, 
Table 1

Comparison of different local searches on instance pmed40-p225

\begin{tabular}{|c|c|c|c|c|c|c|c|}
\hline \multirow[b]{2}{*}{ Local search } & \multicolumn{3}{|c|}{ Deviation (\%) } & \multicolumn{3}{|l|}{ Distance } & \multirow[b]{2}{*}{ Time } \\
\hline & Minimum & Average & Maximum & Minimum & Average & Maximum & \\
\hline LS_FI & 0.28 & 2.45 & 5.61 & 15.00 & 38.23 & 68.00 & 8.72 \\
\hline LS_BI & 0.72 & 3.08 & 5.94 & 20.00 & 42.29 & 65.00 & 6.55 \\
\hline LS_FBI & 0.26 & 2.08 & 4.77 & 11.00 & 36.45 & 67.00 & 5.94 \\
\hline
\end{tabular}

Herrán et al. (2020) extended the benchmark set introducing 72 new test instances generated in the same manner.

In the rest of the section, we first compare different search strategies that may be used to explore interchange neighborhood structure, and then we compare Basic VNS against state-of-the-art methods.

\subsection{Comparison of different local search strategies}

The local search with respect to interchange neighborhood structure may be performed using different search strategies. Let us compare the two usual and the new one:

- First_Improvement (FI) search strategy: as soon as an improving solutions is detected in the neighborhood, it is set as new incumbent solution, that is, the move is made;

- Best_Improvement (BI) search strategy: the best among all improving solution is chosen to be the new incumbent solution;

- Facility_Best_Improvement (FBI) search strategy: the best among all improving moves (if any) that involves exchanging given facility $j \in S$ by another facility $j^{\prime} \notin S$ is executed and the resulting solution is set to be new incumbent solution. Note that BI search strategy differs from this search strategy in the sense that BI looks for the best exchange move with respect to all pairs of facilities $j$ and $j^{\prime}\left(j \in S, j^{\prime} \notin S\right)$, while FBI looks for the best facility to replace certain facility $j$.

Therefore, we distinguish three different local search procedures denoted as LS_FI, LS_BI, and LS_FBI, which use FI, BI, and FBI search strategies, respectively. Note that each of them, after accepting new incumbent solution, proceeds by exploring the interchange neighborhood centered now at this new incumbent solution.

In the first series of experiments, we are interested to detect which of these three local searches exhibit the best performances. For that purpose, on the largest instance pmed $40-\mathrm{p} 225$. A, each local search procedure is run 1000 times, each time starting from a different random solution. Note that we tested the local search methods on entire set of instances, but in order to save the space here we present results on instance pmed $40-\mathrm{p} 225$. A. This instance is of the largest size and reveals the typical performance of local searches. The summarized results are reported in Table 1 and Figure 1. In Table 1, columns 2-4 give the minimum, the average, and the maximum \% deviation from the BKS, respectively, over 1000 runs. Columns 5-7 report the minimum, average, and maximum 

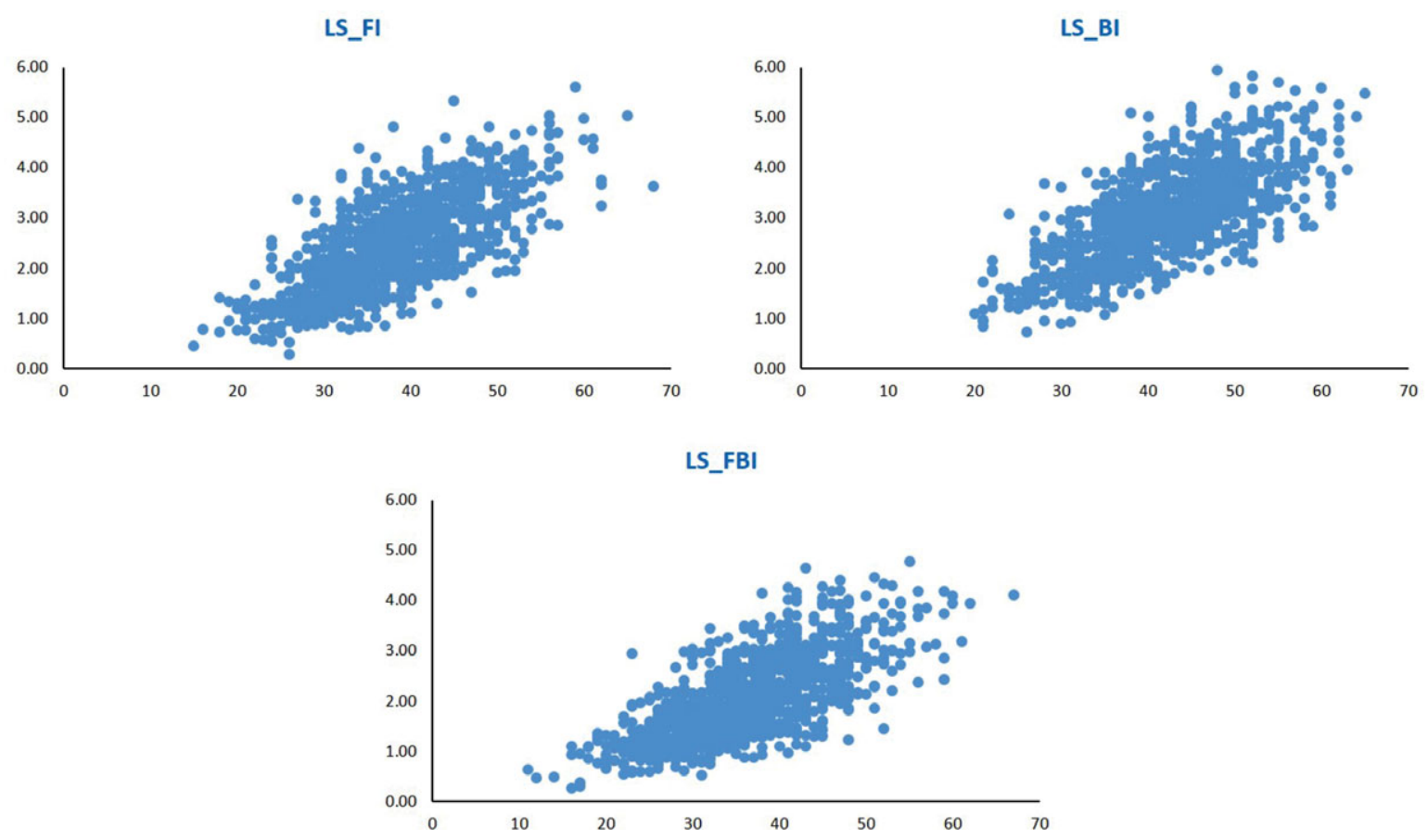

Fig. 1. Distribution of 1000 local maxima on distance-to-target diagram for different local search strategies.

distance between the generated local optima over 1000 runs and the BKS. The distance between solutions $S$ and $S^{\prime}$ is defined as the half of the cardinality of the symmetric difference of sets $S$ and $S^{\prime}$, that is:

$$
d\left(S, S^{\prime}\right)=\frac{\left|S \triangle S^{\prime}\right|}{2}
$$

The last column reports the average computing time spent to reach a local maximum (in seconds). Figure 1 depicts distributions of local optima for these three local search procedures. Distributions are shown on distance-to-target diagrams (Boese et al., 1994), where each local optimum is presented by the point $(x, y)$. Its coordinates are:

(1) $x$ : the distance between the local optimum and the BKS;

(2) $y$ : the percentage deviation of value of the local optimum from the best-known value.

From the reported results, it follows that best performances are exhibited by our new strategy LS_FBI. Local search LS_FI is also able to produce high-quality solutions (of similar quality as those provided by LS_FBI), but it consumes much more CPU time than the other two local searches. Finally, local search LS_BI exhibits the worst performance regarding the solution quality provided. It confirms the observations obtained by comparative analysis on other combinatorial optimization problems (see, e.g., Hansen and Mladenović, 2006): BI is the worst choice if the initial 
solution is taken at random. For the random initial solution, here we show that the semirandom or FBI strategy can be better than FI.

\subsection{Comparison with state-of-the-art methods}

On each test instance, Basic VNS has been executed setting the $t_{\max }$ parameter to 600 seconds. As a local search LS_FBI is used within Basic VNS, since it exhibits the best performances as shown in the previous section. The obtained results are compared with those reported in Herrán et al. (2020). Those results were obtained executing TS algorithm and B\&C algorithm from Belotti et al. (2007) and GRASP heuristic proposed in Colmenar et al. (2016) on the benchmark instances as well as P-VNS (Herrán et al., 2020). Among the compared heuristics, TS, GRASP, and Basic VNS are single-thread heuristics, wh P-VNS executes several threads in parallel. All testings in Herrán et al. (2020) were carried on a machine with an Intel i5 $660 \mathrm{CPU}(3.3 \mathrm{GHz})$ and $8 \mathrm{~GB}$ of RAM, while we used an Intel Xeon E7 $4820 \mathrm{CPU}(2.00 \mathrm{GHz})$. Due to the different computing environments, we have normalized the CPU times reported in Herrán et al. (2020) using the approach described in Dongarra (2014) and data from www.cpubenchmark.net. The normalized CPU times $\bar{T}_{H}$ are computed using the following formula:

$$
T_{H}^{*}=\frac{P(\text { Intel i5 } 660 \mathrm{CPU})}{P(\text { Intel Xeon E7 } 4820 \mathrm{CPU})} T_{H},
$$

where $P($ Intel i5 660 CPU) and $P($ Intel Xeon E7 4820 CPU) are the Passmark CPU scores of the computer used in Herrán et al. (2020) and our computer, respectively, and $T_{H}$ is time reported in Herrán et al. (2020). We assume that the computational power is proportional to the number of cores used, which is used to estimate the Passmark CPU scores of the 2-core machine used in Herrán et al. (2020) and our 10-core machine.

In Tables 2 and 3, we report the results comparison. In both tables, instances are grouped according to the size. In Herrán et al. (2020), instances are grouped in three classes: small instances $(p=n / 16)$, medium $(p=n / 8)$, and large $(p=n / 4)$. Each class contains 48 instances. Our VNS was executed 30 times on each test instance. In each run, we recorded the best solution found as well as the time elapsed upon reaching this solution for the first time (so-called time-to-target). Therefore, for each instance, we stored the best solution value found, the average solution value, and average time-to-target in 30 runs. In Table 2, we report the average of these values over instances within the same class. Column " $\%$ dev." reports the average of the percentage deviations of the best solution values found by our VNS on each instance from the current BKS. On each test instance, the percentage deviation is calculated as

$$
\frac{B K S-V N S_{\text {best }}}{B K S} \times 100 \text {. }
$$

Columns 2-6 in Table 3 provide the number of the current BKS that each method is able to reproduce, whereas the last column "\#new BKS" reports the number of new BKS established by our VNS on each class of instances. In both tables, the last row reports summary results over all instances in the benchmark set. 
Table 2

Comparison with the sate-of-the-art methods

\begin{tabular}{|c|c|c|c|c|c|c|c|c|c|c|c|c|c|}
\hline \multirow[b]{3}{*}{ Instances } & \multirow[b]{3}{*}{$\mathrm{BKS}$} & \multicolumn{2}{|l|}{$\mathrm{B} \& \mathrm{C}$} & \multicolumn{2}{|l|}{$\mathrm{TS}$} & \multicolumn{2}{|c|}{ GRASP } & \multicolumn{2}{|l|}{ P-VNS } & \multicolumn{4}{|l|}{ VNS } \\
\hline & & \multirow[b]{2}{*}{ Value } & \multirow[b]{2}{*}{$T_{H}^{*}$} & \multirow[b]{2}{*}{ Value } & \multirow[b]{2}{*}{$T_{H}^{*}$} & \multirow[b]{2}{*}{ Value } & \multirow[b]{2}{*}{$T_{H}^{*}$} & \multirow[b]{2}{*}{ Value } & \multirow[b]{2}{*}{$T_{H}^{*}$} & \multicolumn{2}{|l|}{ Value } & \multirow{2}{*}{$\begin{array}{l}\text { Average } \\
\text { time }\end{array}$} & \multirow[b]{2}{*}{$\%$ dev. } \\
\hline & & & & & & & & & & Best & Average & & \\
\hline Small & 7582.4 & 7452.0 & 1875.5 & 7463.0 & 283.8 & 7582.4 & 258.1 & 7582.4 & 17.5 & 7582.4 & 7582.2 & 48.1 & 0.00000 \\
\hline Medium & 5856.7 & 5784.8 & 7205.9 & 5814.5 & 413.9 & 5855.5 & 423.0 & 5856.6 & 59.6 & 5856.9 & 5850.4 & 220.1 & -0.00249 \\
\hline Large & 4213.2 & 4090.3 & 7607.5 & 4169.8 & 345.6 & 4205.0 & 669.4 & 4213.1 & 226.9 & 4212.8 & 4202.3 & 329.6 & 0.00961 \\
\hline All & 5884.1 & 5775.7 & 5562.9 & 5815.8 & 347.8 & 5881.0 & 450.1 & 5884.0 & 101.5 & 5884.0 & 5878.3 & 199.3 & 0.00237 \\
\hline
\end{tabular}

Table 3

Comparison with the sate-of-the-art methods (number of BKS)

\begin{tabular}{lcccccc}
\hline Instances & B\&C & TS & GRASP & P-VNS & VNS & “\#new BKS” \\
\hline Small & 9 & 16 & 48 & 48 & 48 & 0 \\
Medium & 6 & 6 & 35 & 43 & 45 & 3 \\
Large & 1 & 1 & 9 & 46 & 40 & 1 \\
All & 16 & 23 & 92 & 137 & 133 & 4 \\
\hline
\end{tabular}

From the reported results, we may infer that the proposed approach yet quite simple clearly outperforms existing single-thread state-of-the-art approaches, that is, TS and GRASP. Our Basic VNS is able to find solutions of higher quality consuming less CPU time than GRASP and TS. In addition, it is quite competitive with the P-VNS, although P-VNS cannot be fairly compared with single-thread approaches since it uses more computational resources. If we compare the number of BKS (see Table 3), we can observe that TS, GRASP, Basic VNS, and P-VNS are able to reproduce 23, 95, 133, and 137 existing BKS, respectively. Moreover, on the medium size instances, our VNS offers more BKS than any method in comparison. In addition, our Basic VNS succeeds to establish four new BKS (three for medium instances and one for a large instance). This further implies that at the moment basic VNS holds 137 BKS, the same number P-VNS held before.

\section{Conclusions}

In this paper, the OpMP has been studied. For solving this NP-hard problem, we have developed a Basic VNS heuristic that follows the principle of the newly proposed "less is more" heuristic approach, that is, it searches for the minimum number of ingredients that enables the proposed heuristic to be more efficient than the current state-of-the-art. More precisely, our basic VNS uses just one neighborhood structure in both shaking and local search procedures. However, although quite simple, the proposed basic VNS outperforms the state-of-the-art results of single-thread heuristics, thus confirming, once again, that sometimes less may yield more. In addition, it is highly competitive with the P-VNS heuristic. 
The future work may include developing "less is more" heuristic approaches for solving other NP-hard optimization problems. Moreover, semirandom local search strategy (FBI), which we proposed here, may be used for solving other discrete optimization problems. In addition, heap data structure, which we introduced to solve facility location problems, can be tried out for solving $p$-median or $p$-center problems and may be compared with those usually used.

\section{Acknowledgments}

This research is partially supported by Serbian Ministry of Education, Science and Technological Development under the grants nos. 174010 and 044006. In addition, this research is partially supported by the framework of the grant number BR05236839 "Development of information technologies and systems for stimulation of personality's sustainable development as one of the bases of development of digital Kazakhstan" and by the National Natural Science Foundation of China (Nos. 71871080, 71601065, 71690235, 71501058), Innovative Research Groups of the National Natural Science Foundation of China (71521001), the Humanities and Social Sciences Foundation of the Chinese Ministry of Education (No. 15YJC630097), and the Base of Introducing Talents of Discipline to Universities for Optimization and Decision-Making in the Manufacturing Process of Complex Product (111 project).

\section{References}

Belotti, P., Labbé, M., Maffioli, F., Ndiaye, M.M., 2007. A branch-and-cut method for the obnoxious $p$-median problem. $4 O R$ 5, 4, 299-314.

Boese, K.D., Kahng, A.B., Muddu, S., 1994. A new adaptive multi-start technique for combinatorial global optimizations. Operations Research Letters 16, 2, 101-113.

Brimberg, J., Mladenović, N., Todosijević, R., Urošević, D., 2017. Less is more: solving the max-mean diversity problem with variable neighborhood search. Information Sciences 382, 179-200.

Brimberg, J., Mladenović, N., Todosijević, R., Urošević, D., 2019. Solving the capacitated clustering problem with variable neighborhood search. Annals of Operations Research 272, 1, 289-321.

Cappanera, P., 1999. A Survey on Obnoxious Facility Location Problems. Dipartimento di Informatica, Università di Pisa.

Chagas, J.B.C., Silveira, U.E.F., Santos, A.G., Souza, M.J.F., 2020. A variable neighborhood search heuristic algorithm for the double vehicle routing problem with multiple stacks. International Transactions in Operational Research 27, $1,112-137$.

Church, R.L., Garfinkel, R.S., 1978. Locating an obnoxious facility on a network. Transportation Science 12, 2, $107-118$.

Colmenar, J.M., Greistorfer, P., Martí, R., Duarte, A., 2016. Advanced greedy randomized adaptive search procedure for the obnoxious p-median problem. European Journal of Operational Research 252, 2, 432-442.

Dongarra, J.J., 2014. Performance of various computers using standard linear equations software. Technical report, CS-89-85, University of Manchester, Manchester, UK.

Duarte, A., Sánchez-Oro, J., Mladenović, N., Todosijević, R., 2018. Variable neighborhood descent. In Martí, R., Pardalos, P.M., Resende, M.G.C. (eds) Handbook of Heuristics. Springer, Basel, Switzerland, pp. 341-367.

Eiselt, H., Laporte, G., 1995. Objectives in location problems. In Drezner, Z. (ed.) Facility Location: a Survey of Applications and Methods. Springer, New York, pp. 151-180.

Gruler, A., Panadero, J., de Armas, J., Prez, J.A.M., Juan, A.A., 2020. A variable neighborhood search simheuristic for the multiperiod inventory routing problem with stochastic demands. International Transactions in Operational Research 27, 1, 314-335.

(C) 2019 The Authors.

International Transactions in Operational Research (C) 2019 International Federation of Operational Research Societies 
Hansen, P., Mladenović, N., 1997. Variable neighborhood search for the p-median. Location Science 5, 4, $207-226$.

Hansen, P., Mladenović, N., 2006. First vs. best improvement: an empirical study. Discrete Applied Mathematics 154, 5, 802-817.

Hansen, P., Mladenović, N., Pérez, J.A.M., 2010. Variable neighbourhood search: methods and applications. Annals of Operations Research 175, 1, 367-407.

Hansen, P., Mladenović, N., Todosijević, R., Hanafi, S., 2017. Variable neighborhood search: basics and variants. EURO Journal on Computational Optimization 5, 3, 423-454.

Herrán, A., Colmenar, J.M., Martí, R., Duarte, A., 2020. A parallel variable neighborhood search approach for the obnoxious p-median problem. International Transactions in Operational Research 27, 1, 336-360.

Irawan, C.A., Imran, A., Luis, M., 2020. Solving the bi-objective capacitated p-median problem with multilevel capacities using compromise programming and VNS. International Transactions in Operational Research 27, 1, 361-380.

Janković, O., Mišković, S., Stanimirović, Z., Todosijević, R., 2017. Novel formulations and VNS-based heuristics for single and multiple allocation p-hub maximal covering problems. Annals of Operations Research 259, 1-2, $191-216$.

Mjirda, A., Todosijević, R., Hanafi, S., Hansen, P., Mladenović, N., 2017. Sequential variable neighborhood descent variants: an empirical study on the traveling salesman problem. International Transactions in Operational Research 24, 3, 615-633.

Mladenović, N., Brimberg, J., Hansen, P., Moreno-Pérez, J.A., 2007. The p-median problem: a survey of metaheuristic approaches. European Journal of Operational Research 179, 3, 927-939.

Mladenović, N., Hansen, P., 1997. Variable neighborhood search. Computers \& Operations Research 24, 11, 1097-1100.

Mladenović, N., Todosijević, R., Urošević, D., 2016. Less is more: basic variable neighborhood search for minimum differential dispersion problem. Information Sciences 326, 160-171.

Pinto, T., Alves, C., Valério de Carvalho, J., 2020. Variable neighborhood search algorithms for the vehicle routing problem with two-dimensional loading constraints and mixed linehauls and backhauls. International Transactions in Operational Research 27, 1, 549-572.

Resende, M.G., Werneck, R.F., 2003. On the implementation of a swap-based local search procedure for the p-median problem. Proceedings of the Fifth Workshop on Algorithm Engineering and Experiments (ALENEX03), pp. 119-127.

Tamir, A., 1991. Obnoxious facility location on graphs. SIAM Journal on Discrete Mathematics 4, 4, $550-567$.

Welch, S., Salhi, S., 1997. The obnoxious p facility network location problem with facility interaction. European Journal of Operational Research 102, 2, 302-319.

\section{Appendix}

Tables A1 and A2 contain detailed results of our VNS on entire set of instances. For each instance, we report the best solution value found, the average solution value, and average time-to-target in 30 runs. In addition, we report the percentage deviation of the best solution value found by VNS from the current BKS for each instance. The percentage deviation is calculated as:

$$
\frac{B K S-V N S_{\text {best }}}{B K S} \times 100 \text {. }
$$

A negative percentage deviation means that a new BKS value is established. All new BKS values are boldfaced as well as corresponding percentage deviations. 
Table A1

Detailed results of VNS on set A instances

\begin{tabular}{|c|c|c|c|c|c|c|c|c|c|c|c|}
\hline \multirow[b]{2}{*}{ Instance } & \multirow[b]{2}{*}{ BKS } & \multicolumn{2}{|c|}{ VNS value } & \multirow{2}{*}{$\begin{array}{l}\text { VNS } \\
\text { time }\end{array}$} & \multirow[b]{2}{*}{$\%$ dev. } & \multirow[b]{2}{*}{ Instance } & \multirow[b]{2}{*}{ BKS } & \multicolumn{2}{|c|}{ VNS value } & \multirow{2}{*}{$\begin{array}{l}\text { VNS } \\
\text { average } \\
\text { time }\end{array}$} & \multirow[b]{2}{*}{$\%$ dev. } \\
\hline & & Best & Average & & & & & Best & Average & & \\
\hline pmed17-p100.A & 4054 & 4054 & 4054.0 & 26.5 & 0.000 & pmed29-p150.A & 4141 & 4141 & 4132.0 & 356.6 & 0.000 \\
\hline pmed17-p25.A & 7317 & 7317 & 7317.0 & 13.3 & 0.000 & pmed29-p37.A & 7404 & 7404 & 7404.0 & 119.6 & 0.000 \\
\hline pmed17-p50.A & 5411 & 5411 & 5410.9 & 210.8 & 0.000 & pmed29-p75.A & 5880 & 5880 & 5774.1 & 232.5 & 0.000 \\
\hline pmed18-p100.A & 4220 & 4220 & 4219.8 & 155.5 & 0.000 & pmed30-p150.A & 4385 & 4385 & 4378.9 & 214.1 & 0.000 \\
\hline pmed18-p25.A & 7432 & 7432 & 7432.0 & 2.1 & 0.000 & pmed30-p37.A & 7704 & 7704 & 7704.0 & 11.2 & 0.000 \\
\hline pmed18-p50.A & 5746 & 5746 & 5746.0 & 25.0 & 0.000 & pmed30-p75.A & 6189 & 6189 & 6183.0 & 287.9 & 0.000 \\
\hline pmed19-p100.A & 4033 & 4033 & 4032.5 & 128.3 & 0.000 & pmed31-p175.A & 4135 & 4135 & 4120.5 & 416.4 & 0.000 \\
\hline pmed19-p25.A & 7020 & 7020 & 7020.0 & 1.6 & 0.000 & pmed31-p43.A & 7424 & 7424 & 7422.0 & 87.9 & 0.000 \\
\hline pmed19-p50.A & 5387 & 5387 & 5386.6 & 94.4 & 0.000 & pmed31-p87.A & 5905 & 5905 & 5905.0 & 182.0 & 0.000 \\
\hline pmed20-p100.A & 4063 & 4063 & 4063.0 & 59.3 & 0.000 & pmed32-p175.A & 4242 & 4242 & 4225.6 & 474.8 & 0.000 \\
\hline pmed20-p25.A & 7648 & 7648 & 7648.0 & 1.2 & 0.000 & pmed32-p43.A & 7794 & 7794 & 7793.0 & 90.3 & 0.000 \\
\hline pmed20-p50.A & 5872 & 5872 & 5872.0 & 21.4 & 0.000 & pmed32-p87.A & 5925 & 5925 & 5918.9 & 234.5 & 0.000 \\
\hline pmed21-p125.A & 4155 & 4155 & 4153.8 & 146.6 & 0.000 & pmed33-p175.A & 4105 & 4105 & 4096.0 & 439.3 & 0.000 \\
\hline pmed21-p31.A & 7304 & 7304 & 7304.0 & 6.8 & 0.000 & pmed33-p43.A & 7598 & 7598 & 7598.0 & 59.1 & 0.000 \\
\hline pmed21-p62.A & 5784 & 5784 & 5782.3 & 242.4 & 0.000 & pmed33-p87.A & 5793 & 5793 & 5789.4 & 335.8 & 0.000 \\
\hline pmed22-p125.A & 4358 & 4358 & 4353.8 & 304.3 & 0.000 & pmed34-p175.A & 4287 & 4287 & 4264.9 & 399.8 & 0.000 \\
\hline pmed22-p31.A & 7900 & 7900 & 7900.0 & 25.2 & 0.000 & pmed34-p43.A & 7725 & 7725 & 7725.0 & 44.6 & 0.000 \\
\hline pmed22-p62.A & 5995 & 5995 & 5994.1 & 90.8 & 0.000 & pmed34-p87.A & 5849 & 5849 & 5841.2 & 294.4 & 0.000 \\
\hline pmed23-p125.A & 4114 & 4114 & 4105.4 & 331.3 & 0.000 & pmed35-p100.A & 5845 & 5845 & 5842.6 & 304.9 & 0.000 \\
\hline pmed23-p31.A & 7841 & 7841 & 7841.0 & 3.4 & 0.000 & pmed35-p200.A & 4007 & 4004 & 3984.6 & 445.2 & 0.075 \\
\hline pmed23-p62.A & 5785 & 5785 & 5784.9 & 138.8 & 0.000 & pmed35-p50.A & 7155 & 7155 & 7154.0 & 164.8 & 0.000 \\
\hline pmed24-p125.A & 4091 & 4091 & 4091.0 & 99.9 & 0.000 & pmed36-p100.A & 6461 & 6461 & 6458.3 & 126.5 & 0.000 \\
\hline pmed24-p31.A & 7425 & 7425 & 7425.0 & 9.5 & 0.000 & pmed36-p200.A & 4319 & 4314 & 4299.0 & 471.0 & 0.116 \\
\hline pmed24-p62.A & 5528 & 5528 & 5527.9 & 127.6 & 0.000 & pmed36-p50.A & 8179 & 8179 & 8178.4 & 95.3 & 0.000 \\
\hline pmed25-p125.A & 4155 & 4155 & 4153.0 & 242.7 & 0.000 & pmed37-p100.A & 6203 & 6203 & 6195.0 & 366.4 & 0.000 \\
\hline pmed25-p31.A & 7552 & 7552 & 7552.0 & 3.1 & 0.000 & pmed37-p200.A & 4593 & 4589 & 4575.5 & 469.4 & 0.087 \\
\hline pmed25-p62.A & 5767 & 5767 & 5767.0 & 177.2 & 0.000 & pmed37-p50.A & 7830 & 7830 & 7829.9 & 260.0 & 0.000 \\
\hline pmed26-p150.A & 4341 & 4339 & 4333.6 & 265.7 & 0.046 & pmed38-p112.A & 5913 & 5915 & 5907.7 & 395.4 & -0.034 \\
\hline pmed26-p37.A & 8112 & 8112 & 8112.0 & 2.6 & 0.000 & pmed38-p225.A & 4428 & 4428 & 4394.1 & 503.2 & 0.000 \\
\hline pmed26-p75.A & 5789 & 5789 & 5787.6 & 247.4 & 0.000 & pmed38-p56.A & 7432 & 7432 & 7432.0 & 81.6 & 0.000 \\
\hline pmed27-p150.A & 4062 & 4062 & 4052.2 & 349.0 & 0.000 & pmed39-p112.A & 5935 & 5935 & 5927.3 & 355.5 & 0.000 \\
\hline pmed27-p37.A & 7556 & 7556 & 7556.0 & 27.9 & 0.000 & pmed39-p225.A & 4369 & 4369 & 4345.0 & 507.7 & 0.000 \\
\hline pmed27-p75.A & 5668 & 5668 & 5664.3 & 199.5 & 0.000 & pmed39-p56.A & 7712 & 7712 & 7712.0 & 61.2 & 0.000 \\
\hline pmed28-p150.A & 4099 & 4099 & 4091.9 & 343.6 & 0.000 & pmed40-p112.A & 6272 & 6272 & 6266.5 & 399.1 & 0.000 \\
\hline pmed28-p37.A & 7366 & 7366 & 7366.0 & 28.6 & 0.000 & pmed40-p225.A & 4571 & 4567 & 4548.8 & 514.1 & 0.088 \\
\hline pmed28-p75.A & 5681 & 5681 & 5675.6 & 242.8 & 0.000 & pmed40-p56.A & 8211 & 8211 & 8209.7 & 133.9 & 0.000 \\
\hline Average & 5795.3 & 5795.3 & 5793.8 & 122.1 & 0.0013 & Average & 5997.8 & 5997.4 & 5987.1 & 276.0 & 0.0092 \\
\hline
\end{tabular}

(c) 2019 The Authors.

International Transactions in Operational Research () 2019 International Federation of Operational Research Societies 
Table A2

Detailed results of VNS on set B instances

\begin{tabular}{|c|c|c|c|c|c|c|c|c|c|c|c|}
\hline \multirow[b]{2}{*}{ Instance } & \multirow[b]{2}{*}{ BKS } & \multicolumn{2}{|c|}{ VNS value } & \multirow{2}{*}{$\begin{array}{l}\text { VNS } \\
\text { time }\end{array}$} & \multirow[b]{2}{*}{$\%$ dev. } & \multirow[b]{2}{*}{ Instance } & \multirow[b]{2}{*}{ BKS } & \multicolumn{2}{|c|}{ VNS value } & \multirow{2}{*}{$\begin{array}{l}\text { VNS } \\
\text { average } \\
\text { time }\end{array}$} & \multirow[b]{2}{*}{$\%$ dev. } \\
\hline & & Best & Average & & & & & Best & Average & & \\
\hline pmed17-p100.B & 3992 & 3992 & 3992.0 & 14.6 & 0.000 & pmed29-p150.B & 4157 & 4157 & 4152.0 & 326.3 & 0.000 \\
\hline pmed17-p25.B & 6905 & 6905 & 6905.0 & 3.1 & 0.000 & pmed29-p37.B & 7529 & 7529 & 7529.0 & 15.1 & 0.000 \\
\hline pmed17-p50.B & 5563 & 5563 & 5563.0 & 88.6 & 0.000 & pmed29-p75.B & 5709 & 5709 & 5707.1 & 264.9 & 0.000 \\
\hline pmed18-p100.B & 4122 & 4122 & 4119.9 & 241.7 & 0.000 & pmed30-p150.B & 4313 & 4313 & 4282.4 & 395.2 & 0.000 \\
\hline pmed18-p25.B & 7662 & 7662 & 7662.0 & 2.0 & 0.000 & pmed30-p37.B & 8048 & 8048 & 8048.0 & 6.1 & 0.000 \\
\hline pmed18-p50.B & 5852 & 5852 & 5852.0 & 15.0 & 0.000 & pmed30-p75.B & 6041 & 6041 & 6039.6 & 166.6 & 0.000 \\
\hline pmed19-p100.B & 4016 & 4016 & 4016.0 & 86.4 & 0.000 & pmed31-p175.B & 4138 & 4138 & 4119.8 & 430.9 & 0.000 \\
\hline pmed19-p25.B & 6816 & 6816 & 6816.0 & 2.6 & 0.000 & pmed31-p43.B & 7320 & 7320 & 7320.0 & 96.4 & 0.000 \\
\hline pmed19-p50.B & 5423 & 5423 & 5423.0 & 23.6 & 0.000 & pmed31-p87.B & 5618 & 5621 & 5614.6 & 303.5 & -0.053 \\
\hline pmed20-p100.B & 4067 & 4067 & 4066.9 & 151.1 & 0.000 & pmed32-p175.B & 4244 & 4242 & 4219.2 & 391.1 & 0.047 \\
\hline pmed20-p25.B & 7349 & 7349 & 7349.0 & 1.3 & 0.000 & pmed32-p43.B & 7899 & 7899 & 7899.0 & 15.7 & 0.000 \\
\hline pmed20-p50.B & 5665 & 5665 & 5665.0 & 24.7 & 0.000 & pmed32-p87.B & 5852 & 5852 & 5824.1 & 435.7 & 0.000 \\
\hline pmed21-p125.B & 4033 & 4033 & 4026.1 & 216.4 & 0.000 & pmed33-p175.B & 4156 & 4156 & 4140.9 & 397.5 & 0.000 \\
\hline pmed21-p31.B & 7331 & 7331 & 7331.0 & 4.8 & 0.000 & pmed33-p43.B & 7611 & 7611 & 7611.0 & 24.3 & 0.000 \\
\hline pmed21-p62.B & 5870 & 5870 & 5870.0 & 57.7 & 0.000 & pmed33-p87.B & 5840 & 5840 & 5835.0 & 265.3 & 0.000 \\
\hline pmed22-p125.B & 4338 & 4338 & 4332.5 & 310.7 & 0.000 & pmed34-p175.B & 4270 & 4270 & 4262.3 & 387.5 & 0.000 \\
\hline pmed22-p31.B & 7695 & 7695 & 7695.0 & 3.2 & 0.000 & pmed34-p43.B & 7514 & 7514 & 7514.0 & 21.9 & 0.000 \\
\hline pmed22-p62.B & 6259 & 6259 & 6259.0 & 26.1 & 0.000 & pmed34-p87.B & 5857 & 5857 & 5856.0 & 342.0 & 0.000 \\
\hline pmed23-p125.B & 4095 & 4095 & 4093.5 & 261.7 & 0.000 & pmed35-p100.B & 5639 & 5639 & 5629.2 & 414.8 & 0.000 \\
\hline pmed23-p31.B & 7137 & 7137 & 7136.9 & 32.5 & 0.000 & pmed35-p200.B & 4109 & 4108 & 4089.8 & 465.8 & 0.024 \\
\hline pmed23-p62.B & 5724 & 5724 & 5724.0 & 94.4 & 0.000 & pmed35-p50.B & 7570 & 7570 & 7570.0 & 54.2 & 0.000 \\
\hline pmed24-p125.B & 4072 & 4072 & 4060.2 & 271.5 & 0.000 & pmed36-p100.B & 6219 & 6219 & 6201.3 & 331.3 & 0.000 \\
\hline pmed24-p31.B & 7190 & 7190 & 7190.0 & 10.1 & 0.000 & pmed36-p200.B & 4319 & 4319 & 4297.1 & 468.4 & 0.000 \\
\hline pmed24-p62.B & 5752 & 5752 & 5751.1 & 141.2 & 0.000 & pmed36-p50.B & 8144 & 8144 & 8144.0 & 56.7 & 0.000 \\
\hline pmed25-p125.B & 4233 & 4233 & 4230.4 & 258.8 & 0.000 & pmed37-p100.B & 6209 & 6211 & 6198.1 & 326.6 & -0.032 \\
\hline pmed25-p31.B & 7552 & 7552 & 7552.0 & 16.8 & 0.000 & pmed37-p200.B & 4609 & 4609 & 4596.2 & 474.1 & 0.000 \\
\hline pmed25-p62.B & 5692 & 5692 & 5690.9 & 180.9 & 0.000 & pmed37-p50.B & 8379 & 8379 & 8379.0 & 25.2 & 0.000 \\
\hline pmed26-p150.B & 4173 & 4173 & 4166.7 & 346.7 & 0.000 & pmed38-p112.B & 5949 & 5949 & 5930.1 & 447.8 & 0.000 \\
\hline pmed26-p37.B & 7643 & 7643 & 7643.0 & 7.4 & 0.000 & pmed38-p225.B & 4446 & 4446 & 4427.7 & 562.2 & 0.000 \\
\hline pmed26-p75.B & 5923 & 5923 & 5923.0 & 132.9 & 0.000 & pmed38-p56.B & 7535 & 7535 & 7534.8 & 218.5 & 0.000 \\
\hline pmed27-p150.B & 4144 & 4144 & 4137.6 & 297.1 & 0.000 & pmed39-p112.B & 6198 & 6198 & 6192.1 & 320.0 & 0.000 \\
\hline pmed27-p37.B & 7448 & 7448 & 7448.0 & 19.9 & 0.000 & pmed39-p225.B & 4266 & 4266 & 4248.3 & 541.0 & 0.000 \\
\hline pmed27-p75.B & 5844 & 5844 & 5843.3 & 156.6 & 0.000 & pmed39-p56.B & 7625 & 7625 & 7624.8 & 142.3 & 0.000 \\
\hline pmed28-p150.B & 4069 & 4069 & 4059.0 & 368.1 & 0.000 & pmed40-p112.B & 6200 & 6200 & 6181.8 & 410.5 & 0.000 \\
\hline pmed28-p37.B & 7388 & 7388 & 7388.0 & 6.8 & 0.000 & pmed40-p225.B & 4524 & 4525 & 4506.3 & 489.3 & -0.022 \\
\hline pmed28-p75.B & 5642 & 5642 & 5637.8 & 261.4 & 0.000 & pmed40-p56.B & 8022 & 8022 & 8021.6 & 188.9 & 0.000 \\
\hline Average & 5741.1 & 5741.1 & 5739.4 & 115.0 & 0.000 & Average & 6002.2 & 6002.3 & 5992.9 & 284.0 & -0.001 \\
\hline
\end{tabular}

(c) 2019 The Authors International Transactions in Operational Research (C) 2019 International Federation of Operational Research Societies 